EMBRYARIDDLE
Aeronautical University

SCHOLARLY COMMONS

\section{International Journal of Aviation,} Aeronautics, and Aerospace

\title{
Numerical Model Derived Altimeter Correction Maps for Non- Standard Atmospheric Temperature and Pressure
}

Thomas A. Guinn

Embry-Riddle Aeronautical University - Daytona Beach, Thomas.Guinn@erau.edu

Frederick R. Mosher

Embry-Riddle Aeronautical University - Daytona Beach, moshe774@erau.edu

Follow this and additional works at: https://commons.erau.edu/ijaaa

Part of the Atmospheric Sciences Commons, Aviation Safety and Security Commons, and the Meteorology Commons

\section{Scholarly Commons Citation}

Guinn, T. A., \& Mosher, F. R. (2015). Numerical Model Derived Altimeter Correction Maps for Non-Standard Atmospheric Temperature and Pressure. International Journal of Aviation, Aeronautics, and Aerospace, 2(2). https://doi.org/10.15394/ijaaa.2015.1060

This Article is brought to you for free and open access by the Journals at Scholarly Commons. It has been accepted for inclusion in International Journal of Aviation, Aeronautics, and Aerospace by an authorized administrator of Scholarly Commons. For more information, please contact commons@erau.edu. 


\section{Numerical Model Derived Altimeter Correction Maps for Non-Standard}

Atmospheric Temperature and Pressure

\section{Cover Page Footnote}

The authors would like to acknowledge the two anonymous reviewers for their careful and thoughtful review of our manuscript. We benefited greatly from their insight. 


\section{Introduction}

It is well known in aviation that both non-standard air temperatures as well as non-standard sea-level pressures have a direct impact on the accuracy of indicated altitude as reported by a pressure altimeter. While correcting for nonstandard sea-level pressure is relatively simple because it only requires knowledge of the pressure at only a single level, correcting for non-standard temperature is much more complex. This is because corrections for temperature require knowledge of the mean temperature of the atmospheric layer between the surface and the aircraft's altitude, a problem that has been documented as far back as the 1920s (Brombacher 1926; Keifer 1936; Meisinger 1920). Since routine upper air atmospheric observations were not widely available at that time, temperature corrections for altitude were based on a variety of different methods to approximate the layer-mean temperature, such as climatological temperature tables (Brombacher, 1926), simple averages of the surface temperature and the temperature at altitude (Brombacher, 1934), and graphical computations using adiabatic diagrams (Keifer 1936).

Currently, the accepted practices for correcting altimeter reading vary depending on the type and stage of flight. For aircraft approach and landing operations, the accepted practice for extreme cold temperature altimeter corrections are determined using surface temperature measurements combined with an assumed linear temperature profile (International Civil Aviation Organization [ICAO], 2006). This practice is used despite the wide availability of high-resolution temperature data both in the vertical and the horizontal that would allow for accurate computations of layer-mean temperatures and, therefore, accurate altimeter corrections. For en route operations in Class A airspace, airline and military transport pilots often use D-values via computer flight plans to provide altitude corrections for temperature. First defined by Bellamy (1945), the $\mathrm{D}$-value provides the difference between the pressure altitude of the aircraft and the true altitude. Therefore, for flights within Class A airspace, the D-value can be added directly to the altimeter's indicated altitude to determine the aircraft's true altitude, which works only because pilots in Class A airspace are required to fly pressure altitudes. Thus, the D-value provides a relatively easy means of obtaining true altitude from indicated for terrain avoidance and performance calculation purposes. For flights below Class A airspace, however, the traditional D-value provides limited useful information because pilots at these altitudes are 
required to set their altimeters to the current altimeter setting, thus adding the Dvalue to the indicated altitude does not result in true altitude. However, numerical model data can be used to create detailed images of current and forecasted altimeter correction data for flights below Class A airspace through use of “corrected” D-value charts.

The primary goal of this paper is to demonstrate a method, using a numerical model output, for creating detailed maps of altimeter corrections for both non-standard temperatures and pressures. This will be accomplished through the development of "corrected" D-values. Corrected D-values are traditional Dvalues corrected for non-standard pressure; thus, they correct both for nonstandard temperatures as well as non-standard sea-level pressure. In short, the corrected D-value provides the difference between the indicated altitude and the true altitude when the altimeter is correctly set to the actual altimeter setting. As with the traditional D-value, the key to calculating corrected D-values is highresolution numerical model data readily available operationally. The paper begins with a background discussion, which includes a brief mathematical review of the role temperature plays in pressure altimetry followed by a more detailed review of two current methods used to correct altitude for cold temperatures. Lastly, we derive and discuss the concept of corrected D-value, including potential uses in operations and education, as well as some of the limitations of the concept.

\section{Background}

\section{The Role of Temperature in Pressure Altimetry}

Understanding the role of temperature in pressure altimetry can best be examined through the hypsometric equation; the derivation of which is well published in numerous meteorology textbooks and presented in the Appendix for completeness. If the secondary of effects of humidity on air density are momentarily neglected, the hypsometric equation (1) states the height difference between two pressure surfaces is dependent only on the mean temperature of the layer. Therefore, a colder layer-mean temperature will result in a smaller height difference between the two pressure surfaces than will a warmer layer.

$h_{2}-h_{1}=\frac{R}{g} \bar{T} \ln \left(\frac{p_{1}}{p_{2}}\right)$ 
The challenge lies in the determination of the mean temperature of the layer. For sufficiently small layers, the mean can be accurately represented with a simple arithmetic mean (2), i.e.,

$\bar{T}=\frac{T_{1}+T_{2}}{2}$,

where $T_{1}$ and $T_{2}$ are the temperatures at two different pressure levels. For relatively large layers, such as between the surface and aircraft altitude, the simple arithmetic mean will likely not be representative of the actual mean temperature of the layer and therefore lead to large errors. This is especially true when large temperature inversions exist between the surface and aircraft altitude. To see this, examine Figure 1. Figure 1a shows the simple layer-mean temperature through a temperature inversion that would result if calculated at only two points, the surface and the aircrafts altitude. In this case, the mean temperature would be identically equal to the surface temperature, completely disregarding the warmer temperatures in the above layer. The result would be a layer-mean temperature significantly colder than the true mean therefore the hypsometric equation would result in an altitude significantly lower than actual. However, if the same inversion was divided into multiple contiguous layers as in Figure $1 \mathrm{~b}$, the simple average temperature of each layer would be significantly more representative of the true average. The total altitude could then be calculated by summing the heights of each individual layer.

Figure 1 also serves to dispel a common misconception with students and even professionals alike (e.g., Graham, 2009) that if the surface temperature and pressure are both standard, then the atmosphere is standard. With regards to temperature, the standard atmosphere not only assumes a surface temperature of $15^{\circ} \mathrm{C}$ but also that the temperature decreases linearly with height at a constant rate of $6.5^{\circ} \mathrm{C} / \mathrm{km}$ (through $0-11 \mathrm{~km}$ ). Clearly with an inversion, even if the surface temperature is standard, the temperature lapse rate would be far from constant. Thus, accurate altitude calculations require detailed knowledge of the entire temperature profile between the aircraft altitude and the ground, such as provided by numerical model output. We will use this approach in the next section when we derive and discuss the corrected D-value. Before proceeding, however, it is enlightening to see the role the hypsometric equation currently plays in 
calculating cold weather altimeter correction tables, such as those available in the Aeronautical Information Manual (AIM) (FAA, 2015). We include the derivation of these tables below to provide a basis for comparison.
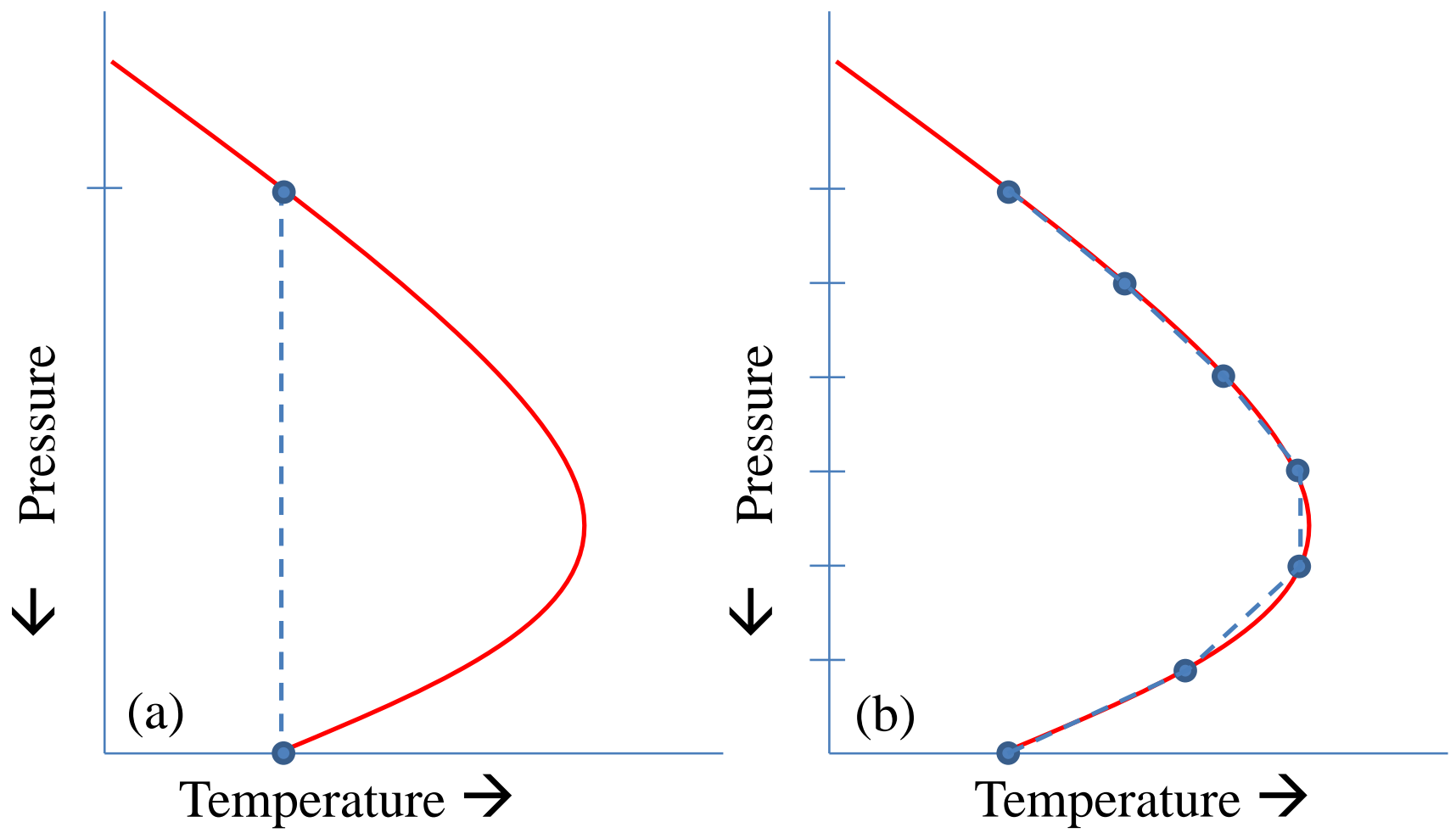

Figure 1. Mean Temperature Determination. This figure illustrates the calculation of a simple arithmetic mean temperature of a temperature profile containing a low-level inversion using a single layer, (a), and multiple layers, (b).

\section{Review of Current Cold Temperature Correction Methods}

The cold-weather altimeter correction can be derived by first defining the relative (i.e., fractional) error $(E)$ between the actual height of a pressure surface above mean sea level $(h)$ and the height of the same pressure surface in the International Standard Atmosphere (ISA) or $h_{I S A}$. This error is defined as:

$E \equiv\left(\frac{h_{I S A}-h}{h}\right)$ 
Since pressure altimeters are calibrated to ISA, $h_{I S A}$, represents the indicated altitude (corrected for mean sea level pressure by setting the Kollsman window to the current altimeter setting) while $h$ represents the true altitude above mean sea level pressure.

Our interest here is relating $E$ to the temperature difference between the ISA and the actual atmosphere. This can be accomplished through the use of the hypsometric equation (1) discussed earlier. Using the hypsometric equation allows the error, $E$, to be expressed in terms of the layer-mean temperature of the atmospheric layer in question and the layer-mean temperature of the same atmospheric layer in the standard atmosphere. Here the height, $h$, is taken to be the height difference $\left(z_{2}-z_{1}\right)$ between a reference pressure, e.g. mean sea level pressure, and the pressure at altitude. Making use of this substitution gives:

$E=\left(\frac{\bar{T}_{I S A}-\bar{T}}{\bar{T}}\right)$,

where $\bar{T}_{I S A}$ is the mean temperature, in Kelvin, of the layer in the standard atmosphere while $\bar{T}$ is the mean temperature, also in Kelvin, of the layer in the actual or observed atmosphere. The constant terms from the hypsometric equation cancel, as do the terms involving pressure.

As mentioned earlier, in the ISA, the temperature decreases linearly with height below $11 \mathrm{~km}(36,000 \mathrm{ft})$ at a rate of $-6.5^{\circ} \mathrm{C} / \mathrm{km}(-1.98 \mathrm{~K} / \mathrm{kft})$. Given a base temperature of $15^{\circ} \mathrm{C}(288.15 \mathrm{~K}), T_{I S A}$ can be expressed as $T_{I S A}=288.15+$ $L z$. Using this, the arithmetic mean temperature of a layer of depth $h$ can be expressed as:

$\bar{T}_{I S A}=288.15+0.5 L h$,

where $\bar{T}_{I S A}$ is in Kelvin, and $L$ is the tropospheric standard temperature lapse rate of $-1.98 \mathrm{~K} / \mathrm{kft}$. Note the standard temperature at mean sea level is assumed to be $288.15 \mathrm{~K}\left(15^{\circ} \mathrm{C}\right)$ regardless of whether or not the sea-level pressure is standard. For a mean sea level pressure deviation from a standard of 1" Hg, this introduces an additional error of less than one percent $(2 \mathrm{~K})$, even for temperatures as cold as $-50^{\circ} \mathrm{C}$. 
A key assumption of the cold weather altimeter correction tables is that the actual environmental lapse rate is also linear and equal to the ISA temperature lapse rate. Using this assumption, the mean temperature of the layer in the actual atmosphere can be expressed as:

$\bar{T}=T_{o}+0.5 L h$,

where $T_{o}$ is the environmental (i.e., the station) temperature at mean sea level in Kelvin. It's important to note the atmospheric temperature rarely decreases at a constant rate, so this method provides only a rough approximation of actual conditions.

Substituting equations (5-6) back into (4) gives an expression for the relative error, $E$, in terms of only the surface temperature $\left(T_{o}\right)$ and the height above mean sea level $(h)$ in $\mathrm{kft}$.

$E \equiv\left(\frac{h_{I S A}-h}{h}\right)=\left(\frac{288.15-T_{o}}{T_{o}+0.5 L h}\right)$

Thus, the necessary amount of altitude correction $(C)$ required by the pilot is simply the altimeter indicated altitude, $h$, times the relative error, $E$, or

$C=h *\left(\frac{288.15-T_{o}}{T_{o}+0.5 L h}\right)$

Note the importance of the sign. When surface temperature is colder than standard, the correction will be positive, meaning the pilot would need to add this height difference to any minimum published height values to ensure safe altitudes. It's also important to note this equation assumes the station is located at mean sea level, which is also rarely the case. However, we can incorporate the effects of station elevation by again assuming a constant temperature lapse rate and using the station elevation $\left(h_{s t n}\right)$. If the temperature (Kelvin) at the station is given as $T_{s t n}$, and the temperature is assumed to increase at a rate of $+1.98 \mathrm{~K} / \mathrm{kft}(-L)$ during descent, the approximate sea-level temperature ( $\left.\widetilde{T}_{o}\right)$ can be expressed as:

$\tilde{T}_{o} \approx T_{s t n}-L h_{s t n}$ 
Table 1.

Altimeter Cold-temperature Correction Values (feet).

\begin{tabular}{|c|c|c|c|c|c|}
\hline \multirow{2}{*}{$\begin{array}{l}\text { Station } \\
\text { Temperature } \\
\left({ }^{0} \mathrm{C}\right)\end{array}$} & \multicolumn{5}{|c|}{$\begin{array}{l}\text { Indicated altitude above station elevation (feet) } \\
\text { (Station Elevation } 0 \text { feet MSL) }\end{array}$} \\
\hline & 500 & 1000 & 1500 & 3000 & 5000 \\
\hline 0 & 30 & 60 & 90 & 170 & 280 \\
\hline-10 & 50 & 100 & 150 & 290 & 490 \\
\hline-20 & 70 & 140 & 210 & 420 & 710 \\
\hline-30 & 100 & 190 & 280 & 570 & 950 \\
\hline-40 & 120 & 240 & 360 & 720 & 1210 \\
\hline-50 & 150 & 300 & 450 & 890 & 1500 \\
\hline \multirow{2}{*}{$\begin{array}{l}\text { Station } \\
\text { Temperature } \\
\left({ }^{\circ} \mathrm{C}\right)\end{array}$} & \multicolumn{5}{|c|}{$\begin{array}{l}\text { Indicated altitude above station elevation (feet) } \\
\text { (Station Elevation 5,000 feet } M S L \text { ) }\end{array}$} \\
\hline & 500 & 1000 & 1500 & 3000 & 5000 \\
\hline 0 & 10 & 20 & 30 & 60 & 100 \\
\hline-10 & 30 & 60 & 90 & 180 & 300 \\
\hline-20 & 60 & 110 & 160 & 310 & 520 \\
\hline-30 & 80 & 150 & 230 & 450 & 760 \\
\hline-40 & 100 & 200 & 300 & 600 & 1010 \\
\hline-50 & 130 & 260 & 390 & 770 & 1300 \\
\hline \multirow{2}{*}{$\begin{array}{l}\text { Station } \\
\text { Temperature } \\
\left({ }^{0} \mathrm{C}\right)\end{array}$} & \multicolumn{5}{|c|}{$\begin{array}{l}\text { Indicated altitude above station elevation (feet) } \\
\text { (Station Elevation 10,000 feet } M S L \text { ) }\end{array}$} \\
\hline & 500 & 1000 & 1500 & 3000 & 5000 \\
\hline 0 & 0 & -10 & -20 & -50 & -90 \\
\hline-10 & 10 & 20 & 30 & 60 & 110 \\
\hline-20 & 40 & 70 & 100 & 190 & 320 \\
\hline-30 & 60 & 110 & 170 & 330 & 550 \\
\hline-40 & 80 & 160 & 240 & 480 & 810 \\
\hline-50 & 110 & 220 & 320 & 650 & 1090 \\
\hline
\end{tabular}


Substituting (8) into (9) while including the height of both the station elevation and the altitude in our computation of the mean temperature, gives an altitude temperature correction $\left(C_{s t n}\right)$ for an altitude, $h$, above the station elevation $h_{s t n}$ of:

$C_{s t n}=h\left[\frac{288.15-\widetilde{T}_{o}}{\tilde{T}_{o}+0.5 L\left(h+h_{s t n}\right)}\right]$.

This is the same equation as provided in ICAO (2006) regarding altimeter temperature corrections. The key difference between equations (8) and (10) is $C_{s t n}$ assumes the station is located above mean sea level; therefore, the two expressions become equivalent when $h_{s t n}$ is zero. It should be noted that the published correction table in the AIM (FAA, 2015). This table was also constructed using (8), thus assuming the station is located at mean sea level. Table 1 provides the altimeter temperature correction $\left(C_{s t n}\right)$ in feet to the indicated altitudes given a temperature and station elevation. The table includes results for three different station elevations, 0, 5,000, and 10,000 ft MSL, respectively.

There are two important results from Table 1. First, the error grows with altitude for all cases. Once again illustrating there is no single additive constant for a given station temperature that works for all altitudes. Second, for a given station temperature, the error is greater for a given altitude above the station if the station elevation is at mean sea level compared to higher station elevations. This is again related to the hypsometric equation and the assumption of a linear temperature profile. Since the temperature is assumed to warm linearly with descent between the station elevation and mean sea level, the mean temperature of the layer will be slightly warmer for the same station temperature when the station is located above mean sea level than if the station were located at mean- sea level. The slightly warmer mean temperatures result in smaller cold-temperature altitude corrections. Thus, the assumption of a station at mean sea level, as published in the AIM (FAA, 2015), provides the most conservative correction.

While this method provides appropriate guidance for obstacle avoidance during approach and landing, it provides little information for cold-temperature corrections for enroute flight operations at altitudes greater than 5,000 ft AGL. 
For en route flights, recommended corrections for cold temperatures are even more simplified. ICAO (2006) recommends prescribed minimum obstacle clearances (MOCs) that are independent of observed temperature. For terrain variations between 3,000-5,000 ft, ICAO (2006) simply recommends a MOC of $1,476 \mathrm{ft}$. While for terrain variations greater than 5,000 ft, ICAO (2006) recommends a MOC of $1,969 \mathrm{ft}$.

In the next section, we develop a new method for cold-temperature correction that can be applied to any altitude and that works as well for general aviation (GA) aircraft below Class A airspace. This method uses numerical-model predicted temperature values to calculate the difference between the true altitude and the indicated altitude and therefore makes no assumption of a constant temperature lapse rate for the observed atmosphere. Therefore, this method provides accurate temperature corrections even when temperature lapse rates deviate significantly from ISA lapse rates.

\section{Derivation of Corrected D-Value}

To derive an expression for the corrected D-value, we again assume the atmosphere to be an ideal gas in hydrostatic balance. The first step is to derive a simple expression for the indicated altitude corrected for non-standard pressure. The derivation begins with the hydrostatic balance equation for an ideal gas, i.e.

$\frac{d p}{d z}=-\frac{g p}{R T}$

Since the temperature lapse rate in the standard atmosphere is defined to be constant, substituting $T=T_{o}+L z$ into (11) gives the following expression.

$\frac{d p}{p}=-\frac{g_{o}}{R} \frac{d z}{\left(T_{o}+L z\right)}$

As before, $L$, is the standard temperature lapse rate, now expressed in SI units, i.e., $-6.5 \mathrm{~K} / 1000 \mathrm{~m}$. Here $R$ is the gas constant for dry air, which is set to $287.053 \mathrm{~J} /(\mathrm{kg} \cdot \mathrm{K})$. Integrating with respect to height from zero to an arbitrary geopotential altitude, $h(p)$, above standard mean sea level pressure, $p_{o}$, while assuming $g$ is constant in the troposphere at $g_{o}=9.80665 \mathrm{~ms}^{-2}$ (National Oceanic and Atmospheric Administration [NOAA], 1976) gives: 
$\frac{p}{p_{o}}=\left(1+\frac{L h}{T_{o}}\right)^{-\frac{g_{o}}{R L}}$.

Solving directly for altitude yields:

$h=\frac{T_{o}}{L}\left[\left(\frac{p}{p_{o}}\right)^{-\frac{R L}{g_{o}}}-1\right]$.

Equation (14) provides an expression for the pressure altitude (PA), which is similar in form to the expression used by the National Weather Service (NOAA, 2015). To obtain the indicated altitude (IA), the PA must be corrected for nonstandard sea-level pressures. The same hydrostatic equation (12) can again be integrated for the height difference between the actual mean sea level pressure $\left(p_{M S L}\right)$ and standard mean sea level pressure $\left(p_{o}\right)$. In the case when mean sea level pressure is greater than standard, the height difference must be added. When the mean sea level pressure is less than standard, the height difference must be subtracted. Combining these results with (14) gives a single expression for the aircraft indicated altitude $\left(h_{I A}\right)$ at an arbitrary pressure level, $p$, i.e.

$h_{I A}=\frac{T_{o}}{L}\left[\left(\frac{p}{p_{o}}\right)^{-\frac{R L}{g_{o}}}-\left(\frac{p_{M S L}}{p_{o}}\right)^{-\frac{R L}{g_{o}}}\right]$.

The next step is to determine the true altitude $\left(h_{T A}\right)$ for comparison with the IA given by (15). For $h_{T A}$, we again use the hypsometric equation; however, we now apply it to the height between two pressure levels, using the simple arithmetic mean temperature of the layer rather than a height-dependent temperature profile. This yields:

$\Delta h=\frac{R}{g_{o}} \bar{T} \ln \left(\frac{p_{b}}{p_{t}}\right)$

where $\Delta h$ is the height difference or thickness between the two pressure surfaces, $p_{t}$ is the top pressure surface, $p_{b}$ is the bottom pressure surface, and $\bar{T}$ is the arithmetic mean of the temperatures at the top and bottom of the layer. To 
calculate the true altitude, we divide the atmosphere into several small layers commensurate with the resolution of the numerical model, and we determine the thickness of each layer using (16). We then obtain the total height by summing the thicknesses of the individual layers. This results in an expression for the true altitude $\left(h_{T A}\right)$ of the atmospheric layer between mean sea level pressure and the pressure sensed at aircraft altitude by the altimeter:

$h_{T A}=-\frac{R}{2 g_{o}} \sum_{n=1}^{N-1}\left[\left(T_{n+1}+T_{n}\right) \ln \left(\frac{p_{n+1}}{p_{n}}\right)\right]$,

where $p_{N}$ and $T_{N}$ are the pressure and temperature, respectively, at altitude $h_{T A}$, while $p_{1}$ and $T_{1}$ are the pressure and temperature, respectively, at mean sea level.

Since both (15) and (17) assume gravity to be constant, they technically result in geopotential altitudes (see Appendix) rather than the desired geometric altitudes. Although the difference is small in the troposphere, additional accuracy can be obtained by converting geopotential height to geometric height using the following conversion from NOAA (1976),

$Z=h \frac{R_{e}}{\left(R_{e}-h\right)}$,

where $Z$ is the geometric height, $h$ is geopotential height, and $R_{e}=6,356,766 \mathrm{~m}$ is the mean radius of the Earth (assumed constant with latitude here). By applying (18) to both (15) and (17), we can now define the corrected D-value, $D_{c}$, as the difference between the geometric true altitude and the geometric IA:

$D_{c}=Z_{T A}-Z_{I A}$.

Atmospheric humidity also has an effect on altitude calculations because it affects the overall density of the air. Though secondary to temperature effects, we can readily incorporate humidity by replacing the actual temperature in equation (18) with the virtual temperature, $T_{v}$. The virtual temperature is the temperature required for dry air to have the same density as air at the same temperature but with nonzero humidity. Since humid air is less dense (lower average molecular weight) than dry air, the virtual temperature is always slightly warmer than the 
actual temperature and therefore causes the layer heights to be slightly larger. For most practical applications, the virtual temperature correction can be approximated by $T_{v} \approx T(1+0.61 w)$, where $T$ is the air temperature in Kelvin and $w$ is the mixing ratio, i.e., the grams of water vapor per gram of dry air (Wallace and Hobbs, 2006). Since the mixing ratio is readily available in numerical model output, the inclusion of humidity effects becomes trivial. Neglecting virtual temperature only results in temperature differences of typically less than $3 \mathrm{~K}(1 \%)$ even in warm tropical atmospheres and less than $0.5 \mathrm{~K}(0.2 \%)$ in cold arctic atmospheres.

\section{Corrected D-Value: Products and Application}

\section{Product Generation}

We now apply equations (15-19) to the numerical model derived temperature, pressure and moisture output from the North American Mesoscale (NAM) model to create graphical displays of corrected D-value, $D_{c}$. The NAM model produces gridded temperature and moisture fields over the U.S. with a horizontal resolution of $12 \mathrm{~km}$ using 60 vertical levels (National Centers for Environmental Prediction [NCEP], 2013). The data is then interpolated to evenly-spaced vertical pressure levels in $25 \mathrm{mb}$ increments, plus mean sea level pressure. The model is run every six hours starting at $00 \mathrm{Z}$ and produces an 84hour forecast in three-hour increments (NCEP, 2013). Results from the model for the $875 \mathrm{mb}, 750 \mathrm{mb}$, and $650 \mathrm{mb}$ pressure levels (corresponding to pressure altitudes of 3,999 ft., $8091 \mathrm{ft}$., and 11,792 ft., respectively) are shown in Figures 2-4. These pressure levels were chosen to be representative of flight altitudes near 4,000 ft., 8,000 ft., and 12,000 ft, respectively. For maximum detail, the products are displayed as images rather than traditional graphics, which use line contours. This enables the user to see a wider range of structure in the data (Mosher, 2015). The yellow, orange, red, and purple colors indicate the air is colder than standard, so the aircraft would be lower than indicated. The black line shows where the indicated altitude equals the true altitude. The green and blue colors show where the air is warmer than the standard atmosphere, so the aircraft would be higher than indicated. 


\section{Potential Operational Applications}

The primary operational use for such products is to provide increased situational awareness and educational training for GA pilots. The graphical displays give pilots an immediate visual indication of the variation in altimeter error with respect to altitude, position, and time. This is also useful for education. While most aviation weather textbooks and materials quantify the error associated with failing to adjust the altimeter for non-standard pressures, their discussions of the effects of non-standard temperature are typically more descriptive rather than quantitative in nature. The errors are usually expressed as a percentage of the increase in the average temperature of layer (Lester, 2001) or estimated in terms of the temperature deviation from standard at a single flight level (FAA, 1975). Graphical images of corrected D-value provide students a more detailed quantification of the direct impacts of temperature on altimeter readings so they can better understand the variation and magnitude of potential errors. Plus, since the model data includes forecasts, the images can also be looped or animated to demonstrate how the temperature error varies with time. Lastly, graphical images of long-term averages could be compiled and animated to demonstrate how the error varies seasonally or monthly.

A potential secondary use for corrected D-value charts could be to provide a measure of the necessary altitude correction to provide safe aircraft separation between aircraft using GPS altimetry and aircraft using pressure altimetry. Currently, altimeter errors due to temperature differences do not create problems for aircraft separation because the FAA requires all aircraft to use pressure altimeters. Therefore, despite all altitudes being in error, they all have nearly identical errors so vertical separation is maintained. However, since the accuracy of GPS altimeters is not sensitive to temperature, separation errors will exist between aircraft using GPS altimeters and those using pressure altimeters. To mitigate this problem, tables of corrected D-values could be created similar to FB Wind bulletins and incorporated into flight planning. This could potentially help ensure all aircraft maintain proper separation. It should be emphasized that at present, this is not an issue because FAA regulations require all aircraft to use pressure altimeters. 


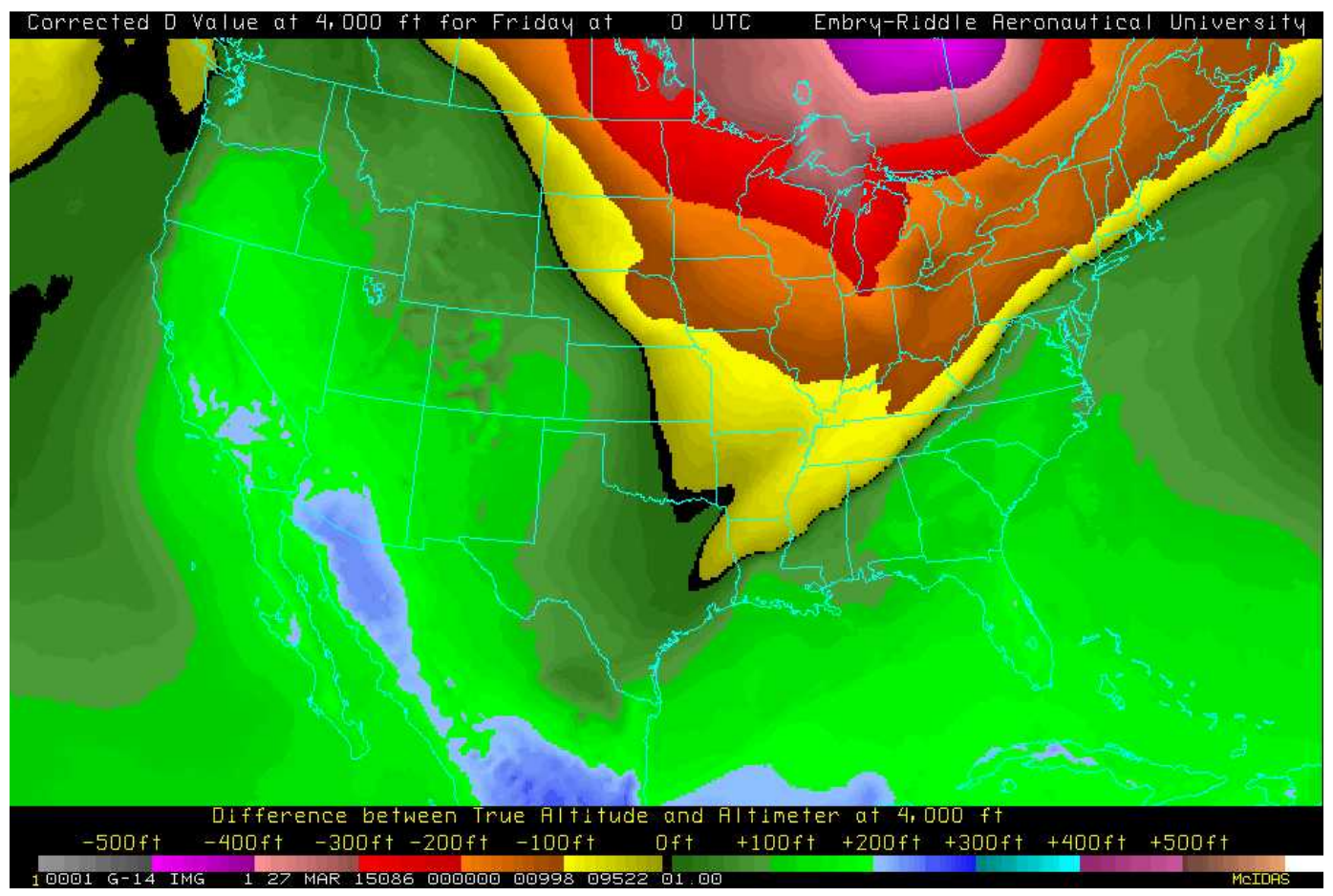

Figure 2. Corrected D-Value forecast for an altitude of approximately $4,000 \mathrm{ft}$ MSL valid on Friday, March 27, 2015 at 00Z. The yellow, orange, red, and purple colors indicate the air is colder than standard, so the aircraft would be lower than indicated. The black line shows where the indicated altitude equals the true altitude. The green and blue colors show where the air is warmer than the standard atmosphere, so the aircraft would be higher than indicated. 


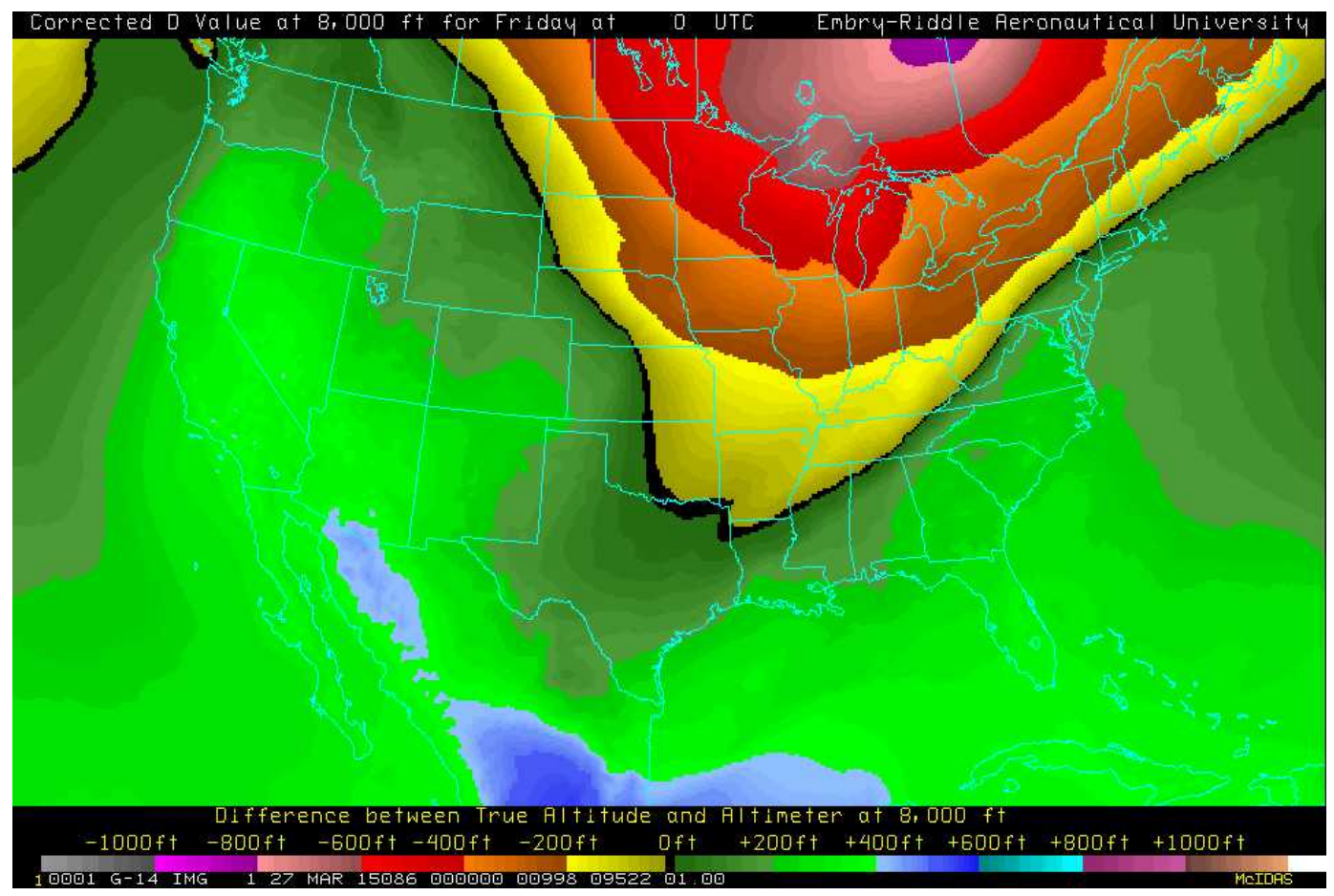

Figure 3. Corrected D-Value forecast for an altitude of approximately 8,000 ft MSL valid on Friday, March 27, 2015 at 00Z. While the colors are the same as Fig. 2, the values of the colors are twice the values of Fig. 2. For example, the red areas in Fig. 2 represent values in the range -200 to $-300 \mathrm{ft}$, while the red colors for Fig. 3 represent values in the range -400 to $-600 \mathrm{ft}$. 


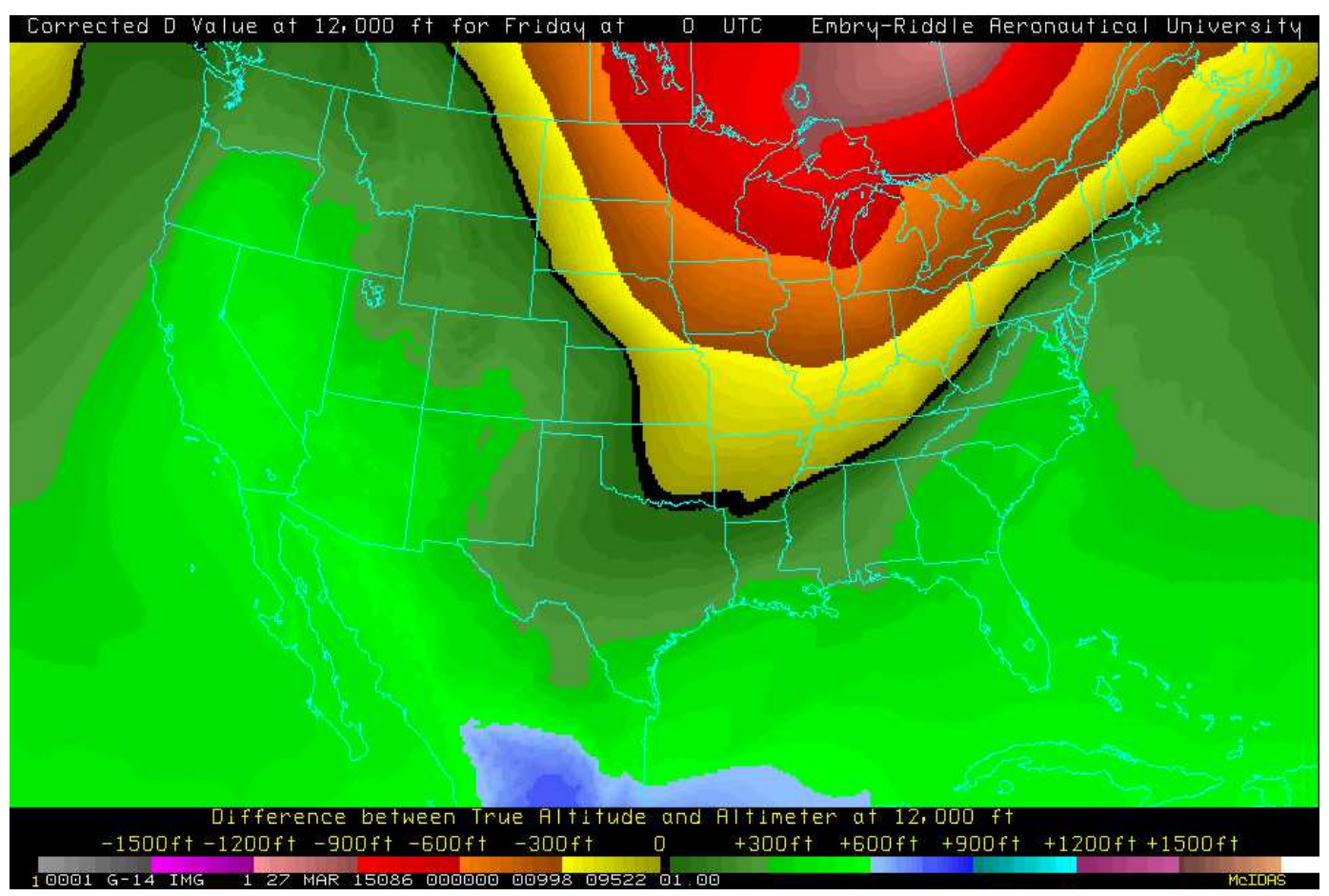

Figure 4. Corrected D-Value forecast for an altitude of approximately $12,000 \mathrm{ft}$ MSL valid on Friday, March 27, 2015 at 00Z. Again the color scheme is the same as in Figs. 2-3, but the values are different. Here the reds represent values in the range -600 to $-900 \mathrm{ft}$, i.e. the aircraft is actually 600 to $900 \mathrm{ft}$ lower than indicated.

\section{Limitations}

One current limitation of using corrected D-value images for operations is the correction is only valid for individual pressure surfaces, not specific height surfaces. For example, the corrected D-value can be applied to the $650 \mathrm{mb}$ pressure surface, which is typically located near an altitude of 12,000 ft above mean sea level. Since the actual $650 \mathrm{mb}$ pressure surface may be higher or lower than $12,000 \mathrm{ft}$ at a location on any given day, the correction represents only an approximation of that required for flights near an indicated altitude of 12,000 ft. Therefore, the pilot must choose the most appropriate pressure level (or pressure altitude) applicable for the flight. To mitigate this problem, charts can be provided at multiple pressure altitudes to give a more detailed depiction of how the correction varies with pressure level. By producing charts or tables in $50 \mathrm{mb}$ 
increments (approximately 1,500 ft intervals) below 18,000 ft, they should have sufficient resolution for providing accurate terrain avoidance guidelines.

\section{Summary and Conclusions}

Non-standard temperatures have a direct effect on the accuracy of altimeter readings, and quantifying the magnitude of the error cannot be accomplished using simple rules of thumb. This is because the error depends on the vertical temperature structure of the atmospheric layer between the surface and the aircraft rather than the temperature at any single level. However, by using a numerical model data output, combined with basic altitude equations, the effects of non-standard temperature on altimeter readings can readily be quantified and visualized. Because the data stems from the numerical model output, not only can current analysis charts be provided, but forecasts of cold-weather impacts can be provided as well. This would provide increased situational awareness for GA pilots, especially during their pre-flight planning stages. Equally important, instructors at aviation schools can now demonstrate the effect of cold temperatures on altimeter corrections with visualizations. These graphics would, therefore, provide both operational and educational benefits.

The corrected D-value forecast products shown here are currently available real-time via the internet at http://fltwx.db.erau.edu/aviationfcst.php. For future work, we plan to create similar graphics using additional pressure levels. In addition, we are investigating the use of archived numerical model data to create seasonal and monthly averages of altimeter corrections to provide educational tools for use by both aviation and meteorology professors. 


\section{References}

Bellamy, J.C. (1945). The Use of Pressure Altitude and Altimeter Correction in Meteorology, Journal of Meteorology, 2, 1-79. http://dx.doi.org/ 10.1175/1520-0469(1945)002<0001:TUOPAA > 2.0.CO;2

Brombacher, W. G. (1926). Compensation of Altimeters and Altigraphs for Air Temperature, Monthly Weather Review, 54, 343. http://dx.doi.org/ 10.1175/1520-0493(1926)54<343a:COAAAF>2.0.CO;2

Brombacher, W.G. (1934). Measurements of Altitude in Blind Flying. Technical Notes National Advisory Committee for Aeronautics No. 503.

FAA. (2015). Aeronautical Information Manual. Washington D.C: Author.

FAA. (1975). AC 00-6A Aviation Weather for Pilots and Flight Operations Personnel. Washington D.C.: Author.

Graham, M. (2009). GPS Altitude. Cross Country, 123.

ICAO. (2006). Document 8168 OPS/611 Aircraft Operations, vol. 1, Flight Procedures, $5^{\text {th }}$ ed., Ch 4, Altimeter Corrections. Retrieved from http://code7700.com/pdfs/ icao_doc_8168_vol_1.pdf

Lester, P. (2001). Aviation Weather (2nd ed.). Englewood, CO: Jeppesen.

Kiefer, P.J. (1936). Determination of Altitude from the Adiabatic Chart and the Refsdal Aerogram, Monthly Weather Review, 64, 69-71. http://dx.doi.org/ 10.1175/1520-0493(1936)64<69:DOAFTA>2.0.CO;2

Meisnger, C. L. (1920). The Effect of Barometric Pressure upon Altimeter Readings, Monthly Weather Review, 48, 529. http://dx.doi.org/ 10.1175/1520-0493(1920)48<529a:TEOBPU>2.0.CO;2

Mosher, Frederick R. (2015). Aviation Weather Forecasts beyond Today. Paper presented at the $17^{\text {th }}$ Conference on Aviation, Range, and Aerospace Meteorology, Phoenix, AZ. 
NCEP. (2013). Product Description Document: NCEP Model Analyses \& Guidance. Retrieved from http://products.weather.gov/PDD/NCEP_PDD_MAG.pdf

NOAA. (1976). U.S. Standard Atmosphere, 1976. Document S/T 76-1562, 241p.

NOAA. (2015). Pressure Altitude. Retrieved March 19, 20015 from http://www.srh.noaa.gov/ images/epz/wxcalc/pressureAltitude.pdf.

Wallace, J.M, \& P.F. Hobbs (2006), Atmospheric Science: An Introductory Survey (2nd ed,). Burlington, MA: Academic Press. 


\section{Appendix}

The derivation of the hypsometric equation relies on the assumption of hydrostatic equilibrium for an ideal gas, which stated simply, implies the vertical pressure gradient force is exactly balanced by the gravitational force. To visualize this, imagine a small rectangular slab of atmosphere of area, $A$, thickness, $\Delta z$, and density, $\rho$, as shown in Fig. 1. Now consider the three fundamental vertical forces acting on the slab. On the top of the slab there is a pressure force exerted by the atmosphere above. This negatively directed (downward) force is simply the atmospheric pressure at the top of the slab times the area of the slab, or stated mathematically, $F_{p t}=-p_{t} \cdot A$. Likewise, the atmosphere below the slab is exerting a positively directed (upward) force, which is simply the atmospheric pressure at the bottom of the slab times the area, or $F_{p b}=+p_{b} \cdot A$. Lastly, the force of gravity is the mass of the slab multiplied by gravity. Since mass is the density times volume, the negatively directed force of gravity can be expressed as $F_{g}=-\rho A \Delta z \cdot g$. For hydrostatic equilibrium balance to exist, these forces must sum to zero, i.e.,

$$
\sum F=F_{p t}+F_{p b}+F_{g}=0
$$

Substituting our expressions from above gives:

$$
-p_{t} \cdot A+p_{b} \cdot A-\rho A \Delta z \cdot g=0
$$

Dividing through by mass (i.e., volume multiplied by density) and rearranging terms provides an expression for hydrostatic balance in terms of force per unit mass.

$$
\frac{p_{t}-p_{b}}{\rho \Delta z}=-g
$$

In the limit as $\Delta z \rightarrow 0$, the expression reduces to the classic hydrostatic equation,

$$
\frac{1}{\rho} \frac{d p}{d z}=-g
$$




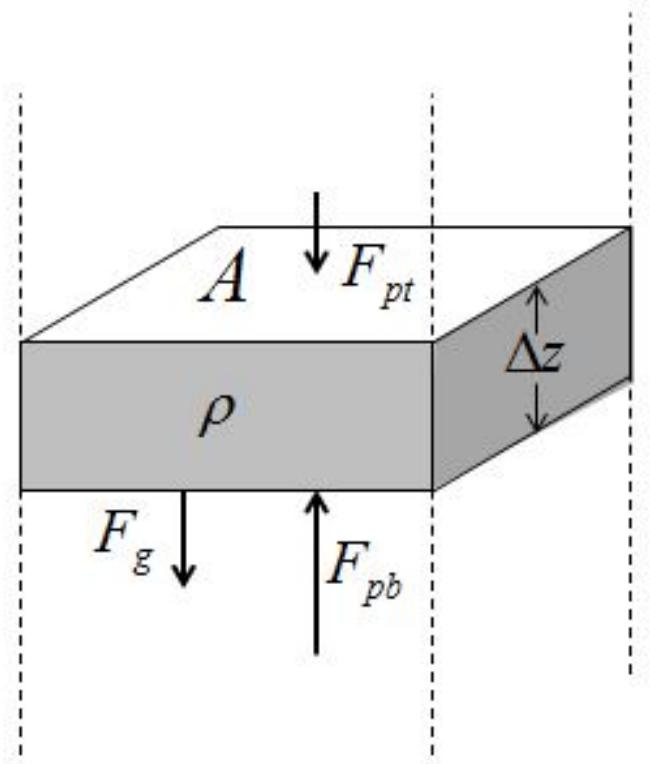

Figure A1. Balance of forces on an idealized slab of air.

While the hydrostatic equation provides a relatively simple relationship between pressure and height, it is difficult to use in practice because it requires knowledge of density. However, because air is an ideal gas, density can be related directly to temperature and pressure, i.e.,

$$
p=\rho R T,
$$

where $R$ is the gas constant for dry air. Substituting into the hydrostatic equation gives

$$
\frac{d p}{p}=-\frac{g}{R} \frac{d z}{T}
$$

This equation can easily be integrated between the heights at two pressure levels, $p_{1}$ and $p_{2}$, i.e.,

$$
\int_{p_{1}}^{p_{2}} d \ln p=-\frac{g_{o}}{R} \int_{h\left(p_{1}\right)}^{h\left(p_{2}\right)} \frac{d z}{T}
$$

Here we have assumed gravity to be a constant with height, in which case the resulting height is the geopotential height. For most aviation applications, and 
especially GA applications, the difference between geopotential altitude and geometric altitude is negligible, less than $20 \mathrm{~m} \mathrm{(0.2 \% )} \mathrm{even} \mathrm{at} \mathrm{the} \mathrm{top} \mathrm{of} \mathrm{the}$ troposphere $(11 \mathrm{~km})$. Using geopotential height leaves temperature as the only remaining variable dependent on height (or pressure). To complete the integration, knowledge of how temperature varies with height (or pressure) is required. Typically, temperature is either assumed to be a linear function of height or a constant, e.g., the mean value for the layer. When working with observed temperature profiles, the layer-mean temperature is most commonly used, which is defined as:

$$
\bar{T}=\frac{\int_{p_{1}}^{p_{2}} T d p}{\int_{p_{1}}^{p_{2}} d p}
$$

Making this substitution gives the desired relationship between height change (thickness) and pressure change, which is known as the hypsometric equation.

$$
h_{2}-h_{1}=\frac{R}{g_{o}} \bar{T} \ln \left(\frac{p_{1}}{p_{2}}\right)
$$

